ERRATUM

\title{
Erratum to: Primary Care Validation of a Single-Question Alcohol Screening Test
}

\author{
Peter C. Smith, MD, MSc ${ }^{7,5}$, Susan M. Schmidt , Donald Allensworth-Davies, MSc ${ }^{2}$, \\ and Richard Saitz, MD, MPH ${ }^{3,4}$
}

\begin{abstract}
'Section of General Internal Medicine, Department of Medicine, Boston Medical Center and Boston University School of Medicine, Boston, MA, USA; ${ }^{2}$ Data Coordinating Center, Boston University School of Public Health, Boston, MA, USA; ${ }^{3}$ Clinical Addiction Research and Education (CARE) Unit, Section of General Internal Medicine, Department of Medicine, Boston Medical Center and Boston University School of Medicine, Boston, MA, USA; ${ }^{4}$ Youth Alcohol Prevention Center and Department of Epidemiology, Boston University School of Public Health, Boston, MA, USA; ${ }^{5}$ Section of General Internal Medicine, Department of Medicine, Boston University School of Medicine, Boston, MA, USA.
\end{abstract}

$\mathrm{J}$ Gen Intern Med 25(4):375

DOI: $10.1007 / \mathrm{s} 11606-010-1255-7$

(c) Society of General Internal Medicine 2010

T $\mathrm{n}$ their article, the abstract incorrectly states "MEASURE-

1 MENTS: Participants were asked the single screening question, "How many times in the past year have you had $\mathrm{X}$ or more drinks in a day?", where $\mathrm{X}$ is 5 for men and 4 for women, and a response of $>1$ is considered positive.

It should state: MEASUREMENTS: Participants were asked the single screening question, "How many times in the past year have you had $\mathrm{X}$ or more drinks in a day?", where $\mathrm{X}$ is 5 for men and 4 for women, and a response of 1 or greater is considered positive.

Corresponding Author: Peter C. Smith, MD, MSc; Section of Genera Internal Medicine, Department of Medicine, Boston University School of Medicine, 2nd Floor, Crosstown Center, 715 Albany Street, Boston, MA 02118, USA (e-mail: peter.smith@bmc.org)

The online version of the original article can be found under $h t t p: / / d x$. doi.org/10.1007/s11606-009-0928-6.

Published online February 2, 2010 